\title{
SQUARE INTEGRABLE REPRESENTATIONS AND A PLANCHEREL THEOREM FOR PARABOLIC SUBGROUPS
}

\author{
BY
}

\author{
FREDERICK W. KEENE
}

\begin{abstract}
Let $G$ be a semisimple Lie group with Iwasawa decomposition $G=K A N$. Let $g_{0}=\mathfrak{f}_{0}+\mathfrak{a}+\mathfrak{n}$ be the corresponding decomposition of the Lie algebra of $G$. Then the nilpotent subgroup $N$ has square integrable representations if and only if the reduced restricted root system is of type $A_{1}$ or $A_{2}$. The Plancherel measure for $N$ can be found explicitly in these cases. We then prove the Plancherel theorem in the $A_{1}$ case for the solvable subgroup $N A$ by combining Mackey's "Little Group" method with an idea due to C. C. Moore: we find an operator $D$, defined on the $C^{\infty}$ functions on $N A$ with compact support, such that

$$
\phi(e)=\int_{(N A)^{\circ}} \operatorname{tr}(D \pi(\phi)) d \mu(\pi)
$$

where $(N A)^{\wedge}$ is the unitary dual, $e$ is the identity, and $\mu$ is the Plancherel measure for $N A$, and $D$ is an unbounded selfadjoint operator. In the $A_{1}$ case, $D$ involves fractional powers of the Laplace operator and hence is not a differential operator.
\end{abstract}

I. Introduction. Let $G$ be a unimodular locally compact group. Then (see [4], [5], [19]) we can define a square integrable representation $\pi$ of $G$ on a Hilbert space $H(\pi)$. If $\pi$ is irreducible and unitary, then $\pi$ is square integrable if there are nonzero vectors $x_{1}$ and $x_{2}$ in $H(\pi)$ such that

$$
\int_{G / Z}\left|\left(\pi(s) x_{1}, x_{2}\right)\right|^{2} d \tilde{\mu}(\tilde{s})<\infty .
$$

Here $Z$ is the center of $G$ and $d \tilde{\mu}(\tilde{s})$ denotes integration over the group $G / Z$ with respect to a Haar measure $\tilde{\mu}$ on $G / Z$. This definition makes sense as the integrand is invariant under translations by elements of $Z$ and hence is actually a function on $G / Z$.

In the following, we look at the nilpotent and solvable subgroups of a semisimple Lie group $G$ appearing in the Iwasawa decomposition $G=K A N$. Using results of Moore and Wolf [14], we find the conditions on $G$ for $N$ to have square integrable representations: this happens precisely when the

Received by the editors November 6, 1974 and, in revised form, July 14, 1976.

AMS (MOS) subject classifications (1970). Primary 22D10, 22E25, 22E45, 43A30, 43 A65.

Key words and phrases. Parabolic subgroup, semisimple Lie group, Plancherel theorem, nonunimodular group, square integrable representations, solvable subgroups of type $A N$.

- American Mathematical Society 1978 
reduced restricted root system of $\mathrm{g}_{0}$ (or of $\mathrm{g}$ ) is of type $A_{1}$ or $A_{2}$. Moore and Wolf have shown further that if $N$ has square integrable representations, these form a subset of $\hat{N}$ on which Plancherel measure is concentrated.

Plancherel measure for $A N$ is concentrated on the set of representations of $A N$ gotten by Mackey's "Little Group" method from the representations of $N$ on which Plancherel measure for $N$ is concentrated (see Mackey [12] and Auslander and Moore [2]). By extending and inducing the representations of $N$ up to $A N$ in the $A_{1}$ case, we are able to find the Plancherel formula. The Plancherel formula for $M N$ is worked out in [20] by J. A. Wolf. Wolf, R. L. Lipsman, and the author have written out the formula for $M A N$ in another paper [21].

This work is in essence part of the author's doctoral dissertation [8] at the University of California, Berkeley. The author would like to take this opportunity to thank Professor Joseph A. Wolf for his patient advice and encouragement. He would also like to thank Professors C. C. Moore, D. S. Drucker, and I. Satake for valuable suggestions in the first part of the paper, and Professor R. L. Lipsman for suggestions in \$III. A preliminary announcement of these results appeared in [9].

II. The existence of the square-integrable representations.

A. Square-integrable representations of a nilpotent Lie group. Let $N$ be a connected simply-connected nilpotent Lie group with Lie algebra $\mathfrak{n}$. Let $Z$ and $z$ be the centers of $N$ and $\mathfrak{n}$ respectively, and let $\mathfrak{n}^{*}, z^{*}$ denote the respective linear duals of $\mathfrak{n}$ and $\mathfrak{z}$. If $f \in \mathfrak{n}^{*}$, we define a bilinear form $b_{f}$ on $\mathfrak{n}$ by

$$
b_{f}(x, y)=f([x, y]),
$$

and note that $b_{f}$ is also well defined on $\mathfrak{n} / \mathfrak{z}$. In fact, if $f \in \mathfrak{z}^{*}$, we can extend $f$ to $\mathfrak{n}$ trivially and define $b_{f}$ on $\mathfrak{n} / \mathfrak{z}$.

Given $b_{f}$, we define

$$
\mathfrak{n}_{f}=\left\{x \in \mathfrak{n} \mid b_{f}(x, y)=0 \text { for all } y \in \mathfrak{n}\right\},
$$

and note that $\operatorname{dim} \mathfrak{n}-\operatorname{dim} \mathfrak{n}_{f}=\operatorname{dim}\left(\operatorname{Ad}^{*}(N) f\right)$ where $\operatorname{Ad}^{*}(N) f$ is the orbit of $f$ under the coadjoint representation of $N$ on $\mathfrak{n}^{*}$. Now, if $\mathfrak{n}_{f}=z$, so that the dimension of the orbit is maximal, then the orbit is determined by $f \mid z$. Suppose $f \in z^{*}$ is chosen and extend trivially to $\mathfrak{n}$ by choosing a basis $\mathscr{B}=\left\{z_{1}, \ldots, z_{k}, y_{k+1}, \ldots, y_{n}\right\}$ for $\mathfrak{n}$ with $\left\{z_{1}, \ldots, z_{k}\right\}$ a basis for $z$ and $f\left(y_{i}\right)=0, i=k+1, \ldots, n$. Choose $x \in \mathfrak{n}$ such that $x \notin z$. Then if $\mathfrak{n}_{f}=\mathfrak{z}$, there is a $y \in \mathfrak{n}$ such that $b_{f}(x, y)=f([x, y]) \neq 0$ and $[x, y]$ has a nontrivial central component in the basis $\mathscr{B}$.

Proposition 1. With the above notation, if there is a noncentral $x \in \mathfrak{n}$ such that $[x, y]$ has no nontrivial central component for all $y \in \mathfrak{n}$, then there is no 
$f \in \mathfrak{n}^{*}$ such that $\operatorname{dim} \mathfrak{n}-\operatorname{dim} \mathfrak{n}_{f}=\operatorname{dim}\left(\operatorname{Ad}^{*}(N) f\right)$ is maximal.

COROLlary 1. With the above notation, if there is an $x \in \mathfrak{n}$ such that $[x$, $\mathfrak{n}] \cap \mathfrak{z}=\{0\}$, then there is no $f \in \mathfrak{n}^{*}$ such that $\operatorname{dim} \mathfrak{n}-\operatorname{dim} \mathfrak{n}_{f}=$ $\operatorname{dim}\left(\operatorname{Ad}^{*}(N) f\right)$ is maximal.

REMARKS. 1. This corollary gives us a necessary condition for $\mathfrak{n}_{f}=\mathfrak{z}$. In the case when $\operatorname{dim} z=1$, the condition is also sufficient.

2. A necessary and sufficient condition for $b_{f}$ to be nondegenerate on $\mathfrak{n} / z$ is $\mathfrak{n}_{f}=\mathfrak{z}$.

COROLlaRY 2. If there is a noncentral $x$ in $\mathfrak{n}$ such that $[x, \mathfrak{n}] \cap \mathfrak{z}=\{0\}$, then, for every $f \in \mathfrak{n}^{*}, b_{f}$ is degenerate on $\mathfrak{n} / \mathfrak{z}$.

Then a result of Moore and Wolf [14] implies

Proposinion 2. $N$ has square integrable representations if and only if there exists an $f \in \mathfrak{z}^{*}$ such that $b_{f}$ is nondegenerate of $\mathfrak{n} / \mathfrak{z}$.

If we choose a volume element $\omega$ for $\mathfrak{n} / \mathfrak{z}$, this condition is equivalent to having the determinant of $b_{f}$ with respect to $\omega$ be nonzero. The square root of this determinant is the Pfaffian, $\operatorname{Pf}(f)$.

Proposition $2^{\prime} . N$ has square integrable representations if and only if there exists an $f \in z^{*}$ such that $\operatorname{Pf}(f) \neq 0$.

B. The nilpotent part of a semisimple Lie group. Let $g_{0}$ be a real semisimple Lie algebra, and fix a Cartan decomposition $g_{0}=\mathfrak{f}_{0}+\mathfrak{p}_{0}$. Let a be a maximal abelian subalgebra of $\mathfrak{p}_{0}, \mathfrak{m}$ the centralizer of $\mathfrak{a}$ in $\mathfrak{f}_{0}$, and $\mathfrak{g}_{c}=\mathfrak{f}_{0}+i \mathfrak{p}_{0}$ the compact real form of $g^{\mathrm{C}}$, the complexification of $g_{0}$. Let $\tau$ be the conjugation with respect to $g_{c}$. Let $B(\cdot, \cdot)$ be the Killing form of $g^{C}$ and hence of $g_{0}$ and $\mathfrak{g}_{c}$. We write

$$
\mathfrak{g}^{0}=\mathfrak{m}+\mathfrak{a}
$$

and for $\alpha: \mathfrak{a} \rightarrow \mathbf{R}$ a linear functional, let

$$
g^{\alpha}=\left\{x \in g_{0} \mid[a, x]=\alpha(a) x \text { for all } a \in \mathfrak{a}\right\} .
$$

$\mathrm{g}^{\alpha}$ is an a-root space of $g_{0}$, and $\alpha$ is an a-root of $g_{0}$. Recall that the $a$-roots are also called restricted roots.

THEOREM 1. Let $\alpha$ be a nonzero a-root. Then $\mathrm{g}^{0}$ acts irreducibly over $\mathbf{R}$ on $\mathrm{g}^{\alpha}$ by the adjoint action.

Proof. First we note that $\mathrm{g}^{0}$ acts over $\mathbf{R}$ on each $\mathrm{g}^{\alpha}$ : Let $x \in \mathfrak{g}^{\alpha}, a \in \mathfrak{a}$, $m \in \mathfrak{m}$. Clearly $\mathfrak{a}$ acts on $\mathfrak{g}^{\alpha}$. To see that $\mathfrak{m}$ acts on $\mathfrak{g}^{\alpha}$, we note that $\operatorname{ad}(m) \cdot x \in \mathfrak{g}^{\alpha}$ : 


$$
\begin{aligned}
{[a, \operatorname{ad}(m) \cdot x] } & =[a,[m, x]]=-[m,[x, a]] \\
& =\operatorname{ad} m([a, x])=\alpha(a) \operatorname{ad}(m) \cdot x .
\end{aligned}
$$

If $\operatorname{dim} g^{\alpha}=1$, we are done as

$$
\left[\mathrm{g}^{0}, x\right] \supset[\mathfrak{a}, x]=\mathbf{R} x=\mathrm{g}^{\alpha} \text { for } x \in \mathrm{g}^{\alpha}, x \neq 0 .
$$

Suppose $\operatorname{dim} \mathrm{g}^{\alpha}>1$. Let $e \neq 0$ be in $\mathrm{g}^{\alpha}$. Then $\tau e \in \mathrm{g}^{-\alpha}$ and $\tau e \neq 0$.

$$
(\alpha(a) \tau x=\tau(\alpha(a) x)=\tau[a, x]=[\tau a, \tau x]=[-a, \tau x]
$$

hence

$$
[a, \tau x]=-\alpha(a) \tau x) \text {.) }
$$

Let $w=[e, \tau e]$, so $w \in \mathfrak{a}$ and $w \neq 0$. Then $\{e, \tau e, w\}$ span a threedimensional simple algebra (a TDS) with maximal torus spanned by $w$. This TDS acts by the adjoint action on the vector space $g^{2 \alpha}+g^{\alpha}+g^{0}+g^{-\alpha}+$ $g^{-2 \alpha}$ with weights $2 \alpha, \alpha, 0,-\alpha,-2 \alpha . g^{0}$ is the zero-weight space and $g^{\alpha}$ is the $\alpha$-weight space. The representation theory of TDS then shows that

$$
(\operatorname{ad} e)\left(\mathrm{g}^{0}\right)=\left[e, \mathrm{~g}^{0}\right]=\mathrm{g}^{\alpha} \text {. }
$$

Hence given any nonzero element of $g^{\alpha}$, we have $\left[g^{0}, e\right]=g^{\alpha}$, so the action of $g^{0}$ is irreducible. Q.E.D.

We have actually shown a stronger result.

COROLlary. Let $\alpha$ be a nonzero a-root, $e \in g^{\alpha}$ with $e \neq 0$. Then $\left[g^{0}, e\right]=g^{\alpha}$.

THEOREM 2. Let $\phi, \psi, \phi+\psi$ be nonzero a-roots with $\phi$ and $\psi$ linearly independent. Then $\left[g^{\phi}, g^{\psi}\right]=g^{\psi+\phi}$.

Proof. Let $\mathfrak{h}$ be the Cartan subalgebra determined by $\mathfrak{m}+\mathfrak{a}$ so $\mathfrak{h}=\mathfrak{h}_{1}+$ $\mathfrak{a}$, where $\mathfrak{h}_{1}$ is a Cartan for $\mathfrak{m}$. Then

$$
\begin{aligned}
& g^{\phi}=\sum\left\{g^{\alpha} \mid \alpha \text { an } \mathfrak{h} \text {-root of } g_{0} \text { such that } \alpha \mid \mathfrak{a}=\phi\right\}, \\
& \mathfrak{g}^{\psi}=\sum\left\{g^{\beta} \mid \beta \text { an } \mathfrak{h} \text {-root of } g_{0} \text { such that } \beta \mid \mathfrak{a}=\psi\right\},
\end{aligned}
$$

and

$$
\mathfrak{g}^{\psi+\phi}=\sum\left\{\mathfrak{g}^{\gamma} \mid \gamma \text { is an } \mathfrak{h} \text {-root of } g_{0} \text { such that } \gamma \mid \mathfrak{a}=\psi+\phi\right\}
$$

Then

$$
\begin{array}{r}
\mathfrak{g}^{\phi+\psi} \supset \sum\left\{\mathfrak{g}^{\alpha+\beta} \mid \alpha \text { an } \mathfrak{h} \text {-root of } g_{0} \text { with }\left.\alpha\right|_{\mathfrak{a}}=\phi,\right. \\
\beta \text { an } \mathfrak{h} \text {-root of } g_{0} \text { with }\left.\beta\right|_{\mathfrak{a}}=\psi, \\
\text { and } \left.\alpha+\beta \text { an } \mathfrak{h} \text {-root of } g_{0}\right\}
\end{array}
$$




$$
\mathfrak{g}^{\phi+\psi} \supset\left[\mathrm{g}^{\phi}, \mathrm{g}^{\psi}\right] \text {. }
$$

We want to show that $\left[g^{\phi}, g^{\psi}\right] \neq\{0\}$. Let $\psi-p \phi, \ldots, \psi+q \phi$ be the $\phi$ string through $\psi$, and $V=g^{\psi+q \phi}+\cdots+g^{\psi+\phi}+g^{\psi}+\cdots+g^{\psi-p \phi}$. Let $e_{\phi} \in \mathfrak{g}^{\phi}, e_{-\phi} \in \mathfrak{g}^{-\phi}$ be nonzero elements. Choose $w=\left[e_{\phi}, e_{-\phi}\right]$ so that $\left\{e_{\phi}\right.$, $\left.e_{-\phi}, w\right\}$ span a TDS. This TDS acts on $V$ by the adjoint action. If we let $\langle\cdot$, - $\rangle$ be the inner product of roots in $a^{*}$, then we know that

$$
2\langle\phi, \psi\rangle /\langle\phi, \phi\rangle=p-q \text {. }
$$

Suppose that

$$
U=\sum_{j=1}^{q} g^{\psi+j \phi}, \quad W=\sum_{j=-p}^{0} g^{\psi+j \phi},
$$

and that $U$ and $W$ are invariant subspaces of $V$ under the action of the TDS. Then $w$ acts with trace 0 on each of these subspaces, so that

$$
0=\sum_{j=1}^{q}\left(\operatorname{dim} g^{\psi+j \phi}\right)(\langle\psi, \phi\rangle+j\langle\phi, \phi\rangle)
$$

and

$$
0=\sum_{j=0}^{p}\left(\operatorname{dim} g^{\psi-j \phi}\right)(\langle\psi, \phi\rangle-j\langle\phi, \phi\rangle) .
$$

But (*) implies $\langle\psi, \phi\rangle\langle 0$, while (**) implies $\langle\psi, \phi\rangle>0$ if $p>0$ and $\langle\psi$, $\phi\rangle=0$ if $p=0$. Thus $U$ and $W$ are not invariant subspaces, and so the map ad $e_{\phi}: g^{\psi} \rightarrow g^{\psi+\phi}$ is nonzero.

Let $e \in g^{\phi}, f \in g^{\psi}$ such that $[e, f] \neq 0$ in $g^{\phi+\psi}$. Then by the corollary to Theorem 1,

$$
\left[\mathrm{g}^{0},[e, f]\right]=\mathrm{g}^{\phi+\psi} \text {. }
$$

But

$$
\begin{aligned}
{\left[g^{0},[e, f]\right] } & =\left\{[w,[e, f]] \mid w \in g^{0}\right\} \\
& =\left\{[w,[e, f]]+[e,[w, f]] \mid w \in g^{0}\right\} \\
& \subset\left[g^{\phi}, f\right]+\left[e, g^{\psi}\right] \subset\left[g^{\phi}, g^{\psi}\right],
\end{aligned}
$$

so $g^{\phi+\psi} \subset\left[g^{\phi}, g^{\psi}\right]$ and hence $g^{\phi+\psi}=\left[g^{\phi}, g^{\psi}\right]$. Q.E.D.

Let $\Sigma_{a}^{+}$be a positive a-root system, and write

$$
\mathfrak{n}=\sum\left\{\mathfrak{g}^{\phi} \mid \phi \in \Sigma_{\mathfrak{a}}^{+}\right\}
$$

for the nilpotent subalgebra in the Iwasawa decomposition of $g_{0}$. We let $z$ be the center of $\mathfrak{n}$ (continuing our notation from above with this particular $\mathfrak{n}$ ), and let $\phi \in \Sigma^{+}$. Then $g^{\phi} \cap z=g^{\phi}$ or $\{0\}$ as $g^{\phi} \cap z$ is invariant under $g^{0}$. (To see that $z$ is invariant under $g^{0}$ let $g \in \mathfrak{g}^{0}, x \in \mathfrak{z}, y \in \mathfrak{n}$. Then 


$$
[y, \operatorname{ad} g \cdot x]=[[y, g], x]+[g,[y, x]]=0
$$

and $x \in z$, so ad $g \cdot x \in z$.)

Thus we have

$$
z=\sum\left\{\left(g^{\phi} \cap z\right) \mid \phi \in \Sigma_{a}^{+}\right\}=\sum\left\{g^{\phi} \mid g^{\phi} \cap z \neq\{0\}\right\} .
$$

If $\phi \in \Sigma_{a}^{+}$is not the maximal a-root, then there is a simple a-root $\psi$ such that $\phi+\psi$ is an a-root.

THEOREM 3. Let $\mu$ be the maximal $a-r o o t$. Then $z=g^{\mu}$.

Proof. Let $\phi \in \Sigma_{a}^{+}$. If $\phi \neq \mu$, then there is a $\psi \in \Sigma_{a}^{+}$such that $\phi+\psi \in$ $\Sigma_{a}^{+}$. Then $\left[g^{\phi}, g^{\psi}\right]=g^{\phi+\psi}$ tells us that $g^{\phi}$ is not contained in the center, so $g^{\phi} \cap z=\{0\}$. Thus $z=g^{\mu}$. Q.E.D.

Proposition 3. Let $\alpha \in \Sigma_{a}^{+}$such that $\alpha$ is orthogonal to the maximal a-root $\mu$. If $x \in \mathfrak{g}^{\alpha}$ and $y \in \mathfrak{n}$, then $[x, y] \in \Sigma\left\{\mathfrak{g}^{\beta} \mid \beta \in \Sigma_{a}^{+}, \beta \neq \mu\right\}$.

Proof. Let $y=\Sigma\left\{y_{\gamma} \mid y_{\gamma} \in \mathfrak{g}^{\gamma}, \gamma \in \Sigma_{\mathfrak{a}}^{+}\right\}$, so $[x, y]=\Sigma\left[x, y_{\gamma}\right]$ where

$$
\begin{aligned}
{\left[x, y_{\gamma}\right] } & \in\{0\} \quad \text { if } \alpha+\gamma \text { is not a nonzero } a \text {-root, } \\
& \in \mathrm{g}^{\alpha+\gamma} \text { if } \alpha+\gamma \text { is a nonzero a-root. }
\end{aligned}
$$

If $\alpha+\gamma$ is a nonzero $a$-root, then $\alpha+\gamma \neq \mu$.

If $\alpha+\gamma=\mu$, then $\gamma=\mu-\alpha$ is a root, and the formula for the length of the strings of roots would dictate that $\alpha$ and $\mu$ were not orthogonal. Q.E.D.

Let $G=K A N$ be the Lie group decomposition corresponding to the Lie algebra decomposition $\mathrm{g}_{0}=\mathfrak{f}_{0}+\mathfrak{a}+\mathfrak{n}$, so $\mathfrak{n}$ is the Lie algebra of $N$. We can combine Proposition 1 with Theorem 3 to find out precisely when $N$ has square integrable representations.

THEOREM 4. $N$ has square integrable representations if and only if, in the extended Dynkin diagram for the reduced restricted root system, the maximal restricted root $\mu$ is connected to every simple restricted root.

Proof. Suppose $\alpha$ is a simple restricted root such that $\alpha$ is not connected to $\mu$ in the extended diagram. Then $\alpha$ is orthogonal to $\mu$, and $\alpha+\mu$ is not a restricted root. Hence $\mu-\alpha$ is not a restricted root. Thus for any restricted root $\beta, \alpha+\beta \neq \mu$. By Theorem $3, z=g^{\mu}$, so Proposition 3 tells us that $\left[g^{\alpha}\right.$, $\mathfrak{n}] \cap \mathfrak{n}=\{0\}$, that is, that there is no central component for $\left[\mathfrak{g}^{\alpha}, \mathfrak{n}\right]$. Thus there is an $x \neq 0$ in $\mathfrak{n}$ such that $[x, \mathfrak{n}] \cap z=\{0\}$, and by Proposition 1 there is no $f \in \mathfrak{n}^{*}$ with $b_{f}$ nondegenerate on $\mathfrak{n} / \mathfrak{z}$. Hence by Proposition $2, N$ has no square integrable representations.

The converse can be shown by explicitly showing that in this case the Pfaffian is nonzero. See Keene [8] for details. Q.E.D.

The only diagrams which fulfill the conditions of Theorem 4 are 
$0 \quad A_{1}$

$\alpha$

and

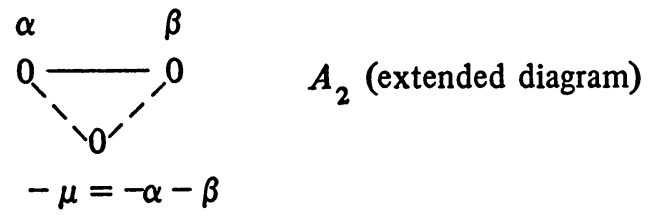

COROLLARY. $N$ has square integrable representations if and only if the extended Dynkin diagram of the reduced restricted root system is of type $A_{1}$ or of type $A_{2}$.

III. The Plancherel formula for $A N$ : The rank 1 case. Let $\mathfrak{g}_{0}=\mathfrak{f}_{0}+\mathfrak{a}+\mathfrak{n}$ be an Iwasawa decomposition of a simple Lie algebra as usual. Suppose the a-root system is of type $A_{1}$. If $G=K A N$ is the corresponding decomposition at the group level, then this condition is equivalent to that of $G / K$ having symmetric space rank 1 . Thus the only algebras $g_{0}$ which fulfill this condition can be realized as so( $1, k ; \mathbf{F})$ for $\mathbf{F}=\mathbf{R}$ (the real numbers), $\mathbf{C}$ (the complex numbers), and $\mathbf{Q}$ (the quaternions), plus the real form of the exceptional Lie algebra of type $F_{4}$ that has maximal compact subalgebra so (9), which corresponds to $\mathcal{C}$, the real Cayley division algebra. Then $\mathfrak{n}$ is given as follows: Let $\mathbf{F}=\mathbf{R}, \mathbf{C}, \mathbf{Q}$, or $\mathcal{C}$. Define a nilpotent Lie algebra $\mathfrak{n}(\mathbf{F})$ by

$$
\mathfrak{n}(\mathbf{F})=\mathbf{F}^{n}+\operatorname{Im} F
$$

where $\operatorname{Im} \mathbf{F}$ is the imaginary part of the real division algebra $\mathbf{F}$, and the multiplication is given, for $x=\left(x_{1}, \ldots, x_{n}\right), y=\left(y_{1}, \ldots, y_{n}\right) \in \mathbf{F}^{n}$, by

$$
[x, y]=\operatorname{Im}\left(\sum_{i=1}^{n} x_{i} \bar{y}_{i}\right)
$$

making $\operatorname{Im} \mathbf{F}$ the center of $\mathfrak{n}(\mathbf{F})$, and, in fact, fulfilling $[\mathfrak{n}(\mathbf{F}), \mathfrak{n}(\mathbf{F})] \subset \mathfrak{z}(\mathfrak{n}(\mathbf{F}))$. Then $\mathfrak{n}=\mathfrak{n}(\mathbf{F})$. When $\mathbf{F}=\mathbf{R}, \mathfrak{n}$ is abelian.

The Lie group $N$ can be written as the manifold $\mathfrak{n}$ with the group multiplication given by the Campbell-Hausdorff formula

$$
\exp (\alpha) \exp (\beta)=\exp \left(\alpha+\beta+\frac{1}{2}[\alpha, \beta]\right)
$$

in this case. Thus if $z, z^{\prime} \in \operatorname{Im}(\mathbf{F})$ and $x, x^{\prime} \in \mathrm{F}^{n}$, the multiplication in $N$ is

$$
(z ; x)\left(z ; x^{\prime}\right)=\left(z+z^{\prime}+\frac{1}{2}\left[x, x^{\prime}\right] ; x+x^{\prime}\right) \text {. }
$$

The Lie group $N A$ is thus the manifold $\mathfrak{n}+\mathfrak{a}$ with the multiplication from the semidirect product: if $a, a^{\prime} \in A$ and $n, n^{\prime} \in N$, then $(n a)\left(n^{\prime} a^{\prime}\right)=n a n^{\prime} a^{-1} a a^{\prime}$, 
so

$$
\begin{aligned}
(z ; x ; a)\left(z^{\prime} ; x^{\prime} ; a^{\prime}\right) & =(z ; x ; 1)(0 ; 0 ; a)\left(z^{\prime} ; x^{\prime} ; a^{\prime}\right)=(z ; x ; 1)\left(a^{2} z^{\prime} ; a x^{\prime} ; a a^{\prime}\right) \\
& =\left(z+a^{2} z^{\prime}+\frac{1}{2}\left[x, a x^{\prime}\right] ; x+a x^{\prime} ; a a^{\prime}\right) .
\end{aligned}
$$

Here $(0 ; 0 ; a)\left(z^{\prime} ; x^{\prime} ; a^{\prime}\right)=\left(a^{2} z ; a x^{\prime} ; a a^{\prime}\right)$ because $z, z^{\prime} \in z(\mathfrak{n})=g^{\mu}$, the maximal root space: if the positive $n$-roots are $\alpha$ and $2 \alpha$, then $z(n)=g^{2 \alpha}$, and $x, x^{\prime} \in g^{\alpha}$. Thus the identity element for $N A$ is $1_{N A}=(0 ; 0 ; 1)$, and $(z ; x$; $a)^{-1}=\left(-a^{-2} z ;-a^{-1} x ; a^{-1}\right)$.

Let $\lambda \in z(\mathfrak{n})^{*}$, the linear dual of $z(\mathfrak{n})$. Let $b_{\lambda}$ be as in II.A.

Definition. A subalgebra $\mathfrak{p}$ of $\mathfrak{n}$ is a (real) polarization at $\lambda$ if $\mathfrak{p}$ is a maximal totally isotropic subspace for $b_{\lambda}$, i.e. if $\lambda([x, \mathfrak{p}])=\{0\}$ if and only if $x \in \mathfrak{p}$.

If $\mathfrak{p}$ is a polarization in $\mathfrak{n}$, we shall denote the corresponding subalgebra $\mathfrak{p}+\{0\} \subset \mathfrak{n}+\mathfrak{a}$ by $\mathfrak{p}$. Similarly the subgroup $\exp (\mathfrak{p})=P \subset N$ shall be regarded as a subgroup of $N A$. Clearly $z(\mathfrak{n}) \subset \mathfrak{p}$. If $\lambda$ is extended trivially to $\mathfrak{p}$, then we can define a character $\chi_{\lambda}$ of $P$ (i.e. a one-dimensional representation of $P$ ) by $\chi_{\lambda}(z ; x ; 1)=e^{i \lambda(z)}$. The Kirillov theory [10] says that every irreducible unitary representation $\xi_{\lambda}$ of $N$ is induced by a representation of the form $\chi_{\lambda}$, that is, $\xi_{\lambda}=\operatorname{Ind}_{P \uparrow N}\left(\chi_{\lambda}\right)$.

TheOREM. Let $\mathfrak{n}=\operatorname{Im}(\mathbf{F})+\mathbf{F}^{n}, \mathbf{F}=\mathbf{R}, \mathbf{C}, \mathbf{Q}$, or $\mathcal{C}$, be a nilpotent Lie algebra as defined above. Let $\mathfrak{a}=\mathbf{R}$ be the (vector) abelian Lie algebra. Let $N A$ be the semidirect product of the corresponding connected simply-connected Lie groups. Let $Z \simeq \mathbf{R}^{k}$ be the center of $N$ and let $D$ be the pseudo-differential operator on $Z$ given by

$$
D=2(2 \pi)^{-q} \Delta^{q / 2}
$$

where $q=n$ if $\mathbf{F}=\mathbf{R}$ and $q=(n(k+1)+2 k) / 2$ if $\mathbf{F} \neq \mathbf{R}$, and where $\Delta$ is the Laplace operator on $Z$. View $D$ as an operator on $N A$, and let $\phi \in$ $C_{c}^{\infty}(N A)$. Then

(1) $D \phi \in L_{1}(N A)$, so $\pi_{\lambda}(D \phi)$ is defined for $\lambda \in z(\mathfrak{n})^{*}$.

(2) If $\lambda \in z(\mathfrak{n})^{*}$ then $\pi_{\lambda}(D \phi)$ is trace class.

(3) trace $\pi_{\lambda}(D \phi)$ is a $C^{\infty}$ function of $\lambda$.

(4) $\phi\left(1_{N A}\right)=\int_{S^{k-1}}$ trace $\pi_{\lambda}(D \phi) d \sigma(\lambda)$, where $d \sigma(\lambda)$ is an appropriate multiple of Lebesgue measure on the unit sphere $S^{k-1}$ in $Z$.

REMARK. (1) and (2) are shown in [21], so here we need prove only (3) and (4).

Proof. We prove the theorem for the case $\mathbf{F}=\mathbf{Q}$; the complex and Cayley cases are identical and the real case similar.

In the quaternionic case, the center $z(\mathfrak{n})=\operatorname{Im}(\mathbf{Q})$ is of dimension 3. Write $\mathbf{Q}=\mathbf{R}^{4}$ and $\operatorname{Im}(\mathbf{Q})=\mathbf{R}^{3}$ as vector spaces. An element of $N A$ will be of the 
form $(z ; x ; a)=\left(z_{1}, z_{2}, z_{3} ; x_{0}, x_{1}, x_{2}, x_{3} ; a\right)$ where $z_{1}, z_{2}, z_{3} \in \mathbf{R} ; x_{0}, x_{1}, x_{2}$, $x_{3} \in \mathbf{R}^{n}$; and $a \in \mathbf{R}^{+}$. The multiplication is given above.

We choose $\lambda: \operatorname{Im}(\mathbf{Q}) \rightarrow \mathbf{R}$ by $\lambda\left(z_{1}, z_{2}, z_{3}\right)=\mu z_{1}, \mu \in R$, so that $\lambda$ corresponds to $l=(\mu, 0,0)$ under the natural pairing from the real inner product $\langle\cdot, \cdot\rangle$. Let $\mathfrak{p}$ be the polarization in $\mathfrak{n}+\mathfrak{a}$ consisting of elements of the form $\left(z ; x_{0}, 0, x_{2}, 0 ; 0\right)$, so $[p, p]$ consists of elements of the form $\left(0,-\left\langle x_{0}\right.\right.$, $\left.\left.x_{2}^{\prime}\right\rangle+\left\langle x_{0}^{\prime}, x_{2}\right\rangle, 0 ; 0,0,0,0 ; 0\right)$ and $\lambda([\mathfrak{p}, \mathfrak{p}])=0$. The group $P \subset N A$ consists of elements of the form $\left(z ; x_{0}, 0, x_{2}, 0 ; 1\right)$ and the character corresponding to $\lambda$ is

$$
x_{\lambda}:\left(z ; x_{0}, 0, x_{2}, 0 ; 1\right) \rightarrow e^{i \lambda(z)}=e^{i\langle l, z\rangle}=e^{i \mu z_{1}} .
$$

(Note that our choice of $\mathfrak{p}$ depends on $\lambda$.) Then $\pi_{\lambda}=\operatorname{Ind}_{P \uparrow N A}\left(\chi_{\lambda}\right)$ represents $N A$ on square integrable functions of $\left(x_{1}, x_{3}, a\right), x_{1}, x_{3} \in \mathbf{R}^{n}, a \in \mathbf{R}^{+}$:

$$
\begin{aligned}
{\left[\pi_{\lambda}\left(z^{\prime} ; x^{\prime} ; a^{\prime}\right) f\right]\left(x_{1}, x_{3}, a\right) } & \\
& =f\left(\left(0 ; 0, x_{1}, 0, x_{3} ; a\right)\left(z^{\prime} ; x_{0}^{\prime}, x_{1}^{\prime}, x_{2}^{\prime}, x_{3}^{\prime} ; a^{\prime}\right)\right) \\
& =f\left(\left(a^{2} z^{\prime}+\frac{1}{2}\left[x, a x^{\prime}\right] ; a x_{0}^{\prime}, x_{1}+a x_{1}^{\prime}, a x_{2}^{\prime}, x_{3}+a x_{3}^{\prime} ; a a^{\prime}\right)\right)
\end{aligned}
$$

where

$$
\begin{aligned}
& a^{2} z^{\prime}+\frac{1}{2}\left[x, a x^{\prime}\right]=\left(a^{2} z_{1}^{\prime}+\frac{1}{2} a\left\langle x_{1}, x_{0}^{\prime}\right\rangle+\frac{1}{2} a\left\langle x_{3}, x_{2}^{\prime}\right\rangle,\right. \\
& a^{2} z_{2}^{\prime}+\frac{1}{2} a\left\langle x_{1}, x_{0}^{\prime}\right\rangle-\frac{1}{2} a\left\langle x_{3}, x_{1}\right\rangle, \\
& \left.a^{2} z_{3}^{\prime}-\frac{1}{2} a\left\langle x_{1}, x_{2}^{\prime}\right\rangle+\frac{1}{2} a\left\langle x_{3}, x_{0}^{\prime}\right\rangle\right) .
\end{aligned}
$$

Letting $(\tilde{z} ; \tilde{x} ; 1) \in P$, we get

$$
\begin{aligned}
{\left[\pi_{\lambda}\left(z^{\prime} ; x^{\prime} ; a^{\prime}\right) f\right]\left(x_{1}, x_{3}, a\right) } & =f\left((\tilde{z} ; \tilde{x} ; 1)\left(0 ; 0, x_{1}+a x_{1}^{\prime}, 0, x_{3}+a x_{3}^{\prime} ; a a^{\prime}\right)\right) \\
& =\chi_{\lambda}(\tilde{z} ; \tilde{x} ; 1) f\left(x_{1}+a x_{1}^{\prime}, x_{3}+a x_{3}^{\prime} a a^{\prime}\right),
\end{aligned}
$$

where

$$
\begin{aligned}
\tilde{z}=\left(a^{2} z_{1}^{\prime}\right. & +a\left\langle x_{1}, x_{0}^{\prime}\right\rangle+a\left\langle x_{3}, x_{2}^{\prime}\right\rangle+\frac{1}{2} a^{2}\left\langle x_{0}^{\prime}, x_{1}^{\prime}\right\rangle+\frac{1}{2} a^{2}\left\langle x_{2}^{\prime}, x_{3}^{\prime}\right\rangle, \\
& a^{2} z_{2}^{\prime}+\frac{1}{2} a\left\langle x_{1}, x_{3}^{\prime}\right\rangle-\frac{1}{2} a\left\langle x_{3}, x_{1}^{\prime}\right\rangle, \\
& \left.a^{2} z_{3}^{\prime}-a\left\langle x_{1}, x_{2}^{\prime}\right\rangle+a\left\langle x_{3}, x_{0}^{\prime}\right\rangle+\frac{1}{2} a^{2}\left\langle x_{0}^{\prime}, x_{3}^{\prime}\right\rangle-\frac{1}{2} a^{2}\left\langle x_{1}^{\prime}, x_{2}^{\prime}\right\rangle\right)
\end{aligned}
$$

and

$$
\tilde{x}=\left(a x_{0}^{\prime}, 0, a x_{2}^{\prime}, 0\right) .
$$

Now we want $\pi_{\lambda}$ of a function applied to $f$, so let $\psi \in D C_{c}^{\infty}(N A)$ where $D$ is defined below:

$$
\begin{aligned}
& {\left[\pi_{\lambda}(\psi) f\right]\left(x_{1}, x_{3}, a\right)} \\
& \quad=\int_{N A} \psi\left(z^{\prime} ; x^{\prime} ; a^{\prime}\right)\left[\pi_{\lambda}\left(z^{\prime} ; x^{\prime} ; a^{\prime}\right) f\right]\left(x_{1}, x_{3}, a\right)\left(a^{\prime}\right)^{r} d a^{\prime} d z^{\prime} d x^{\prime},
\end{aligned}
$$


where $\left(a^{\prime}\right)^{r} d a^{\prime} d z^{\prime} d x^{\prime}$ is right Haar measure on $N A$; the value of $r$ will not matter. This can be rewritten as a multiple integral. Define

$$
\begin{aligned}
F\left(x_{1}, x_{3}, a ; x_{1}^{\prime}, x_{3}^{\prime}, a^{\prime}\right) \\
\quad=\int_{\mathbf{R}^{n}} \int_{\mathbf{R}^{n}} \int_{\mathbf{R}^{3}} \psi\left(z^{\prime} ; x^{\prime} ; a^{\prime}\right) x_{\lambda}(\tilde{z} ; \tilde{x} ; 1) d z^{\prime} d x_{0}^{\prime} d x_{2}^{\prime} \\
\quad=\alpha\left[\psi\left(\cdot ; \cdot, x_{1}^{\prime}, \cdot, x_{3}^{\prime} ; a^{\prime}\right)\right]^{\wedge}\left(a^{2} \mu, \mu\left(a x_{1}+\frac{1}{2} a^{2} x_{1}^{\prime}\right), \mu\left(a x_{3}+\frac{1}{2} a^{2} x_{3}^{\prime}\right)\right),
\end{aligned}
$$

where $\alpha=(2 \pi)^{(2 n+3) / 2}$ and $\left[\psi\left(\cdot ; \cdot, x_{1}^{\prime}, \cdot, x_{3}^{\prime} ; a^{\prime}\right)\right]^{\wedge}$ is the Fourier transform in $2 n+3$ variables, parametrized by $\left(x_{1}^{\prime}, x_{3}^{\prime}, a^{\prime}\right)$. Thus

$$
\begin{aligned}
& {\left[\pi_{\lambda}(\psi) f\right]\left(x_{1}, x_{3}, a\right)=\int_{\mathbf{R}^{n}} \int_{\mathbf{R}^{n}} \int_{\mathbf{R}^{+}} F\left(x_{1}, x_{3}, a ; x_{1}^{\prime}, x_{3}^{\prime}, a^{\prime}\right) } \\
& \cdot f\left(x_{1}+a x_{1}^{\prime}, x_{3}+a x_{3}^{\prime}, a a^{\prime}\right)\left(a^{\prime}\right)^{r} d a^{\prime} d x_{1}^{\prime} d x_{3}^{\prime} \\
&=\int_{\mathbf{R}^{n}} \int_{\mathbf{R}^{n}} \int_{\mathbf{R}^{+}} F\left(x_{1}, x_{3}, a ;\right.\left.\frac{1}{a}\left(x_{1}^{\prime \prime}-x_{1}\right), \frac{1}{a}\left(x_{3}^{\prime \prime}-x_{3}\right), \frac{a^{\prime \prime}}{a}\right) \\
& \cdot f\left(x_{1}^{\prime \prime}, x_{3}^{\prime \prime}, a^{\prime \prime}\right) a^{-2 n-1} \cdot\left(a^{\prime \prime} / a\right)^{r} d a^{\prime \prime} d x_{1}^{\prime \prime} d x_{3}^{\prime \prime},
\end{aligned}
$$

where $x_{1}^{\prime \prime}=x_{1}+a x_{1}^{\prime}, x_{3}^{\prime \prime}=x_{3}+a x_{3}^{\prime}$, and $a^{\prime \prime}=a a^{\prime}$. Let

$$
\begin{aligned}
K\left(x_{1}, x_{3}, a ; x_{1}^{\prime \prime}, x_{3}^{\prime \prime}, a^{\prime \prime}\right) & \\
& =F\left(x_{1}, x_{3}, a, a^{-1}\left(x_{1}^{\prime \prime}-x_{1}\right), a^{-1}\left(x_{3}^{\prime \prime}-x_{3}\right), a^{\prime \prime} / a\right) a^{-2 n-1}\left(a^{\prime \prime} / a\right)^{r},
\end{aligned}
$$

so that

$$
\begin{aligned}
& {\left[\pi_{\lambda}(\psi) f\right]\left(x_{1}, x_{3}, a\right)} \\
& \quad=\int_{\mathbf{R}^{n}} \int_{\mathbf{R}^{n}} \int_{\mathbf{R}^{+}} K\left(x_{1}, x_{3}, a ; x_{1}^{\prime \prime}, x_{3}^{\prime \prime}, a^{\prime \prime}\right) f\left(x_{1}^{\prime \prime}, x_{3}^{\prime \prime}, a^{\prime \prime}\right) d a^{\prime \prime} d x_{1}^{\prime \prime} d x_{3}^{\prime \prime},
\end{aligned}
$$

and the trace of the representation is found by integrating the kernel $K$ along the "diagonal" $\left(x_{1}, x_{3}, a\right)=\left(x_{1}^{\prime \prime}, x_{3}^{\prime \prime}, a^{\prime \prime}\right)$,

$$
\text { trace } \begin{aligned}
\pi_{\lambda}(\psi) & =\int_{\mathbf{R}^{2 n}} \int_{\mathbf{R}^{+}} K\left(x_{1}, x_{3}, a ; x_{1}, x_{3}, a\right) d a d x_{1} d x_{3} \\
& =\int_{\mathbf{R}^{2 n}} \int_{\mathbf{R}^{+}} F\left(x_{1}, x_{3}, a ; 0,0,1\right) a^{-(2 n+1)} d a d x_{1} d x_{3}
\end{aligned}
$$

and $a=a^{\prime \prime}$ implies $a^{\prime}=1$, so the value of $r$ in the right Haar measure makes no difference. Again changing variables, we can rewrite the trace as (with $\left.w=(2 \pi)^{1 / 2}\right)$ 


$$
\begin{aligned}
\operatorname{trace} \pi_{\lambda}(\psi)=2^{2 n+3} \int_{\mathbf{R}^{2 n}} \int_{0}^{\infty}[\psi(\cdot ; \cdot, 0, \cdot, 0 ; 1)]^{\wedge}\left(a^{2} \mu, \mu a x_{1}, \mu a x_{3}\right) & \cdot a^{-(2 n+1)} d a d x_{1} d x_{3} \\
= & w^{2 n+3} \int_{\mathbf{R}^{2 n}} \int_{0}^{\infty}[\psi(\cdot ; \cdot, 0, \cdot, 0 ; 1)]^{\wedge}\left(a^{2} \mu, x_{1}, x_{3}\right) a^{-(4 n+1)} \mu^{-2 n} d a d x_{1} d x_{3} \\
= & \frac{w^{2 n+3}}{2} \int_{\mathbf{R}^{2 n}} \int_{0}^{\infty}[\psi(\cdot ; \cdot, 0, \cdot, 0 ; 1)]^{\wedge}\left(b \mu, x_{1}, x_{3}\right)|b \mu|^{-2 n} b^{-1} d b d x_{1} d x_{3} \\
= & \frac{w^{4 n+3}}{2} \int_{0}^{\infty}[\psi(\cdot ; 0 ; 1)]^{\wedge}(b \mu)|b \mu|^{-2 n} b^{-1} d b \\
= & \frac{w^{4 n+3}}{2} \int_{0}^{\infty}[\psi(\cdot ; 0 ; 1)]^{\wedge}\left(b \frac{\lambda}{\|\lambda\|}\right) b^{-(2 n+1)} d b .
\end{aligned}
$$

We note that this expression is independent of the length of $\lambda$, as it must be since $\pi_{\lambda} \approx \pi_{a \lambda}, a \in A$. We now observe that the vector $\lambda=(\mu, 0,0) \in \mathbf{R}^{3}$ can be rotated without changing the expression. Indeed, by embedding $N A$ in a minimal parabolic subgroup of $\operatorname{Sp}(n, 1)$, we can find a group $M_{0} \simeq \operatorname{Sp}(1)$ that acts on $N A$, commutes with $A$, and rotates $Z$. Then, for any $\lambda \in \mathbf{R}^{3}-$ $\{0\}$, we choose $m \in M_{0}$ such that $\lambda=m \cdot \lambda_{\mu}$ where $\lambda_{\mu}=(\mu, 0,0), \mu \neq 0$, and compute

$$
\text { trace } \begin{aligned}
\pi_{\lambda}(\psi) & =\operatorname{trace} \pi_{m \cdot \lambda_{\mu}}(\psi)=\operatorname{trace} \pi_{\lambda_{\mu}}\left(\psi^{m}\right) \\
& =\frac{w^{4 n+3}}{2} \int_{0}^{\infty}\left[\psi^{m}(\cdot ; 0,1)\right]^{\wedge}\left(b \frac{\mu}{|\mu|}\right) b^{-(2 n+1)} d b \\
& =\frac{w^{4 n+3}}{2} \int_{0}^{\infty}[\psi(\cdot ; 0 ; 1)]^{\wedge}\left(b \frac{\lambda}{\|\lambda\|}\right) b^{-(2 n+1)} d b
\end{aligned}
$$

We show elsewhere [21] that, for $\psi$ of the form $D \phi, D$ defined below, this integral converges absolutely.

On $\mathbf{R}^{3}$ we now take the Laplace operator

$$
\Delta=\sum_{i=1}^{3} \frac{\partial^{2}}{\partial x_{i}^{2}}
$$

and note that we have the equation

$$
(-\Delta \phi)^{\wedge}(x)=|x|^{2} \hat{\phi}(x), \quad \phi \in C^{\infty}\left(\mathbf{R}^{3}\right)
$$

We then define the operator $(-\Delta)^{k}$ by

$$
\left[(-\Delta)^{k} \phi\right](x)=|x|^{2 k} \hat{\phi}(x), \quad k>0 .
$$

Finally we define the operator $D$ on $N A$ by

$$
D=2 w^{-4 n-6}(-\Delta)^{(2 n+3) / 2} \text { in the direction of } Z \text {. }
$$

We take Lebesgue measure $d \sigma(\lambda)$ on $S^{2}$ normalized so that 


$$
\int_{\mathbf{R}^{3}} d z=\int_{0}^{\infty} \int_{S^{2}} b^{2} d \sigma(\lambda) d b
$$

Then we compute

$$
\begin{aligned}
\int_{S^{2}} \operatorname{trace} & \pi_{\lambda}(D \phi) d \sigma(\lambda) \\
& =\int_{S^{2}} \frac{w^{4 n+3}}{2} \int_{0}^{\infty}[D \phi(\cdot ; 0 ; 1)]^{\wedge}(b \lambda) b^{-(2 n+1)} d b d \sigma(\lambda) \\
& =\int_{S^{2}} w^{-3} \int_{0}^{\infty} \hat{\phi}(\cdot ; 0 ; 1)(b \lambda) b^{2} d b d \sigma(\lambda) \\
& =w^{-3} \int_{\mathbf{R}^{3}} \hat{\phi}(\nu ; 0 ; 1) d \nu=\phi(0 ; 0 ; 1)=\phi\left(1_{N A}\right) . \quad \text { Q.E.D. }
\end{aligned}
$$

\section{BIBLIOGRAPHY}

1. S. Araki, On root systems and an infinitesimal classification of irreducible symmetric spaces, J. Math. Osaka City Univ. 13 (1962), 1-34. MR 27 \#3743.

2. L. Auslander and C. C. Moore, Unitary representations of solvable Lie groups, Mem. Amer. Math. Soc. No. 62 (1966). MR 34 \#7723.

3. D. S. Drucker, Non-associative algebras and hermitian symmetric spaces, Doctoral Dissertation, Univ. of California, Berkeley, 1973.

4. R. Godement, Sur les relations d'orthogonalite de V. Bargmann. I, II, C. R. Acad. Sci. Paris 225 (1947), 521-523, 657-659. MR 9, 134.

5. Harish-Chandra, Representations of semisimple Lie groups. VI, Amer. J. Math. 78 (1956), 564-628. MR 18, 490.

6. __ Harmonic analysis on semisimple Lie groups, Bull. Amer. Math. Soc. 76 (1970), 529-551. MR 41 \# 1933.

7. S. Helgason, Differential geometry and symmetric spaces, Academic Press, New York, 1962. MR 26 \#2986.

8. F. W. Keene, Square-integrable representations of Iwasawa subgroups of a semisimple Lie group, Doctoral Dissertation, Univ. of California, Berkeley, 1974.

9.,$L_{2}$-representations and a Plancherel-type theorem for parabolic subgroups, Bull. Amer. Math. Soc. 81 (1975), 117-120. MR 50 \#7415.

10. A. A. Kirillov, Unitary representations of nilpotent Lie groups, Uspehi Mat. Nauk 17 (1962), no. 4 (106), 57-110 = Russian Math. Surveys 17 (1962), 53-104. MR 25 \#5396.

11. B. Kostant, On the existence and irreducibility of certain series of representations, Publ. 1971 Summer School in Math. (I. M. Gel'fand, editor), Bolyai-Janos Math. Soc., Budapest (to appear).

12. G. W. Mackey, Unitary representations of group extensions, I, Acta Math. 99 (1958), 265-311. MR 20 \#4789.

13. C. C. Moore, Representations of solvable and nilpotent groups and harmonic analysis on nil and solvmanifolds, Proc. Sympos. Pure Math., vol. 26, Amer. Math. Soc., Providence, R. I., 1973, pp. 3-44. MR 52 \#5871.

14. C. C. Moore and J. A. Wolf, Square integrable representations of nilpotent groups, Trans. Amer. Math. Soc. 185 (1973), 445-462 (1974). MR 49 \#3033.

15. S. R. Quint, Representations of solvable Lie groups, Lecture Notes, Univ. of California, Berkeley, 1972.

16. I. E. Segal, An extension of Plancherel's formula to separable unimodular groups, Ann. of Math. (2) 52 (1950), 272-292. MR 12, 157.

17. G. B. Seligman, Topics in Lie algebras, Yale Univ. mimeographed notes, 1969; revised, with additions, 1973. 
18. E. M. Stein, Singular integrals and differentiability properties of functions, Princeton Univ. Press, Princeton, N. J., 1970. MR 44 \# 7280.

19. J. A. Wolf, Unitary representations on partially holomorphic cohomology spaces, Mem. Amer. Math. Soc. No. 138 (1974).

20. , Representations of certain semidirect product groups, J. Functional Analysis 19 (1975), 339-372.

21. F. W. Keene, R. L. Lipsman and J. A. Wolf, The Plancherel formula for minimal parabolic subgroups of rank one simple Lie groups, Israel J. Math. (to appear).

Departiment of Mathematics, University of Miami, Coral Gables, Florma 33124

Current address: Department of Mathematics, California State College, San Bernardino, California 92407 